\title{
Spectral theory and operator ergodic theory on super-reflexive Banach spaces
}

\author{
by \\ EARL BERKSON (Urbana, IL)
}

\begin{abstract}
On reflexive spaces trigonometrically well-bounded operators have an operator-ergodic-theory characterization as the invertible operators $U$ such that

$$
\sup _{n \in \mathbb{N}, z \in \mathbb{T}}\left\|\sum_{0<|k| \leq n}\left(1-\frac{|k|}{n+1}\right) k^{-1} z^{k} U^{k}\right\|<\infty .
$$

Trigonometrically well-bounded operators permeate many settings of modern analysis, and this note highlights the advances in both their spectral theory and operator ergodic theory made possible by a recent rekindling of interest in the R. C. James inequalities for super-reflexive spaces. When the James inequalities are combined with Young-Stieltjes integration for the spaces $V_{p}(\mathbb{T})$ of functions having bounded $p$-variation, it transpires that every trigonometrically well-bounded operator on a super-reflexive space $X$ has a norm-continuous $V_{p}(\mathbb{T})$-functional calculus for a range of values of $p>1$, and we investigate the ways this outcome logically simplifies and simultaneously expands the structure theory, Fourier analysis, and operator ergodic theory of trigonometrically well-bounded operators on $X$. In particular, on a super-reflexive space $X$ (but not on a general relexive space) a theorem of Tauberian type holds: the $(C, 1)$ averages in $(*)$ corresponding to a trigonometrically well-bounded operator $U$ can be replaced by the set of all the rotated ergodic Hilbert averages of $U$, which, in fact, is a precompact set relative to the strong operator topology. This circle of ideas is facilitated by the development of a convergence theorem for nets of spectral integrals of $V_{p}(\mathbb{T})$-functions. In the Hilbert space setting we apply the foregoing to the operator-weighted shifts which are known to provide a universal model for trigonometrically well-bounded operators on Hilbert space.
\end{abstract}

1. Introduction and notation. The set of positive integers, the set of all integers, the real line, and the complex plane will be denoted by $\mathbb{N}, \mathbb{Z}$, $\mathbb{R}$, and $\mathbb{C}$, respectively. The unit circle $\{z \in \mathbb{C}:|z|=1\}$ will be designated by $\mathbb{T}$. The symbol " $K$ " with a (possibly empty) set of subscripts will be used to denote a constant which depends only on its subscripts, and which can change in value from one occurrence to another. Except where other-

2010 Mathematics Subject Classification: Primary 26A45, 46B20, 47A35, 47B40.

Key words and phrases: ergodic Hilbert transform, super-reflexive Banach space, spectral decomposition, $p$-variation, trigonometrically well-bounded operator. 
wise indicated, the convergence of a bilateral series $\sum_{k=-\infty}^{\infty} a_{k}$ will mean the convergence of its sequence of bilateral partial sums $\left\{\sum_{k=-n}^{n} a_{k}\right\}_{n=1}^{\infty}$. Throughout all that follows, $\mathfrak{X}$ will be an arbitrary Banach space, and we shall symbolize by $\mathfrak{B}(\mathfrak{X})$ the Banach algebra of all continuous linear operators mapping $\mathfrak{X}$ into $\mathfrak{X}$, the identity operator on $\mathfrak{X}$ being denoted by $I$. A trigonometric polynomial will be a linear combination of a finite subset of the functions $\mathfrak{e}_{n}(z) \equiv z^{n}(z \in \mathbb{T}, n \in \mathbb{Z})$. Given a trigonometric polynomial $Q(z) \equiv \sum_{n} a_{n} z^{n}$ and an invertible $T \in \mathfrak{B}(\mathfrak{X})$, we shall denote by $Q(T)$ the operator $\sum_{n} a_{n} T^{n}$.

Deferring the precise details from spectral theory to 2 , we use this introductory section to fix some notation and to outline our considerations, beginning with the abstract notions of spectral decomposability and spectral integration. An operator $U \in \mathfrak{B}(\mathfrak{X})$ is said to be trigonometrically wellbounded ([5]) provided that $U$ has a "unitary-like" spectral representation

$$
U=\int_{0-}^{2 \pi} e^{i t} d E(t),
$$

where $E(\cdot): \mathbb{R} \rightarrow \mathfrak{B}(\mathfrak{X})$ is a bounded idempotent-valued function possessing certain additional properties reminiscent of, but weaker than, those that would be inherited from a countably additive Borel spectral measure in $\mathbb{R}$, and where the integral in (1.1) is a Riemann-Stieltjes integral existing in the strong operator topology. After suitable normalization, the idempotentvalued function $E(\cdot)$ in 1.1 is uniquely determined, and is called the spectral decomposition of $U$. The spectral decomposition $E(\cdot)$ gives rise to a notion of Riemann-Stieltjes spectral integration against the integrator $E(\cdot)$. Spectral integration with respect to $E(\cdot)$ provides the trigonometrically well-bounded operator $U$ with a norm-continuous functional calculus implemented by $\mathrm{BV}(\mathbb{T})$, the Banach algebra of all complex-valued functions $\psi$ on $\mathbb{T}$ having bounded variation and furnished with the $\mathrm{BV}([0,2 \pi])$-norm of the corresponding function $\psi^{\dagger}(\cdot) \equiv \psi\left(e^{i(\cdot)}\right)$.

Trigonometrically well-bounded operators abound in the structures of modern analysis that require weakened forms of orthogonality to treat delicate convergence phenomena beyond the reach of the unconditional convergence associated with spectral measures. For a variety of naturally occurring examples of trigonometrically well-bounded operators, see, e.g., [8], $\S 4$ of [10], and [20]. In particular, if $\mathfrak{X}$ is a UMD space, then any invertible $U \in \mathfrak{B}(\mathfrak{X})$ such that $U$ is power-bounded (that is, $\sup _{n \in \mathbb{Z}}\left\|U^{n}\right\|<\infty$ ) is trigonometrically well-bounded. For some applications of trigonometrically well-bounded operators to operator ergodic theory and transference methods, see [3], [13], 14], [15], [17], and [18.

Our starting point for this article is the following operator-ergodic-theory characterization of trigonometrically well-bounded operators on an arbitrary 
reflexive Banach space $\mathfrak{X}_{0}$ (see the equivalence of conditions (i) and (ii) of Theorem (2.4) in [6]).

Proposition 1.1. Let $\mathfrak{X}_{0}$ be a reflexive Banach space, and let $U \in$ $\mathfrak{B}\left(\mathfrak{X}_{0}\right)$ be an invertible operator. Then $U$ is trigonometrically well-bounded if and only if

$$
\sup \left\{\left\|\sum_{0<|k| \leq n}\left(1-\frac{|k|}{n+1}\right) \frac{z^{k}}{k} U^{k}\right\|: n \in \mathbb{N}, z \in \mathbb{T}\right\}<\infty .
$$

This article features results in both spectral theory and operator ergodic theory made possible by a recent renewal of interest in the consequences of R. C. James' inequalities for super-reflexive Banach spaces. (For these inequalities, see [30] for the basic notions and fundamental features of super-reflexive spaces, see [31] as well as the celebrated result of P. Enflo in [26], which characterizes super-reflexivity as the property of having an equivalent uniformly convex norm.) When the James inequalities from [30] are combined with Young's inequalities in [40] for the spaces of functions having bounded $p$-variation on the circle (the $V_{p}(\mathbb{T})$ spaces), $1<p<\infty$, it transpires that for every trigonometrically well-bounded operator on a super-reflexive Banach space, spectral integration against its spectral decomposition extends its $\mathrm{BV}(\mathbb{T})$-functional calculus to a norm-continuous $V_{p}(\mathbb{T})$-functional calculus, for a suitable range of values of $p>1$ (Theorem 3.7 below). One indicator of the scope of this extension is that, in contrast to $\mathrm{BV}(\mathbb{T})$, every class $V_{p}(\mathbb{T})$ contains a continuous, nowhere differentiable function of Hardy-Weierstrass type (see Remark 2.8(ii) below).

The spectral integration of function classes of "higher variation" was initiated in [11], but heretofore has been confined to integrating against the spectral decompositions of: invertible power-bounded operators on classical UMD spaces [19], or invertible operators that are separation-preserving and modulus mean-bounded on reflexive Lebesgue spaces of sigma-finite measures [18. Consequently, the results below ensuring spectral integration of $V_{p}(\mathbb{T})$ in the wide setting of super-reflexive spaces markedly expand the scope of spectral integration. Since functions of higher variation act as Fourier multipliers in classical unweighted settings as well as in classical weighted settings (see, e.g., Theorem 8 of [18], Théorème 1 and Lemme 3 of [24]), the spectral integration of the spaces $V_{p}(\mathbb{T})$ provided by Theorem 3.7 below can be viewed as a mechanism for the transference to super-reflexive spaces of a wide family of classical Fourier multipliers, with ramifications for the Fourier analysis of operators. In this regard let us recall that in various contexts where the left bilateral shift is a trigonometrically well-bounded operator (with spectral decomposition $\mathcal{E}(\cdot)$, say) on a sequence space, any bounded complex-valued function $f$ which is continuous a.e. on the circle, and 
such that the spectral integral $\int_{[0,2 \pi]} f\left(e^{i t}\right) d \mathcal{E}(t)$ exists, will act as a Fourier multiplier for the given sequence space, with $\int_{[0,2 \pi]} f\left(e^{i t}\right) d \mathcal{E}(t)$ serving as the multiplier transform of $f$ (p. 16 of [9], Scholium (5.13) of [10], Theorem 4.3 of [16]). Theorem 5.5 below illustrates this point with a new application.

By drawing on $\$ 3$, the treatment in $\$ 4$ furnishes a number of pleasant consequences for the operator ergodic theory of trigonometrically well-bounded operators that logically simplifies and expands their machinery in the superreflexive space setting. In particular, if $U$ is a trigonometrically well-bounded operator on a super-reflexive space $X$, then a Tauberian-type theorem holds (Theorem 4.3 below). Specifically, the $(C, 1)$ averages appearing in the uniform boundedness condition (1.2) can be replaced by the rotated ergodic Hilbert averages of $U$ :

$$
\widetilde{\mathcal{W}}=\left\{\sum_{0<|k| \leq n} \frac{z^{k}}{k} U^{k}: n \in \mathbb{N}, z \in \mathbb{T}\right\} .
$$

In fact, the set $\widetilde{\mathcal{W}}$ is precompact relative to $\sigma_{X}$, the strong operator topology of $\mathfrak{B}(X)$. In the general reflexive space setting, this norm-boundedness of $\mathcal{W}$ need not hold for a trigonometrically well-bounded operator $U$ (see Remark 2.5 below). However, thanks to Hardy's Tauberian Theorem (see, e.g., Theorem II.2.2 in [32]), in the general Banach space setting the set $\widetilde{\mathcal{W}}$ corresponding to a power-bounded trigonometrically well-bounded operator is normbounded (Theorem (3.21) of [7]). So the streamlining effect of Theorem 4.3 below is that for boundedness of $\widetilde{\mathcal{W}}$, the hypothesis of power-boundedness can be dropped provided the underlying Banach space is super-reflexive. In the realm of Fourier analysis of operators on super-reflexive spaces, this streamlining effect is illustrated below by the strong convergence of the operator-valued "Fourier series" associated with a trigonometrically wellbounded operator $U$ and $\mathrm{BV}(\mathbb{T})$-functions (Theorem 4.4). (In this setting, it is further shown that the operator-valued "Fourier series" associated with a trigonometrically well-bounded operator $U$ and $V_{p}(\mathbb{T})$-functions converge $(C, 1)$ in the strong operator topology (Theorem 4.5 below).) The foregoing circle of ideas is facilitated by the development of a suitable convergence theorem for the spectral integrals of $V_{p}(\mathbb{T})$-functions (Theorem 3.9 below).

Since, when taken as a whole, the foregoing results can fail to hold in the general reflexive space setting, it is a pleasant surprise to find them valid throughout the broad context furnished by super-reflexive spaces, which include the UMD spaces ([1], [34]) properly ([22], [35]). In $\$ 5$, we confine attention to the Hilbert space context by taking up some applications of the foregoing to operator-weighted shifts, which have been shown in [16] to furnish a universal model for estimates regarding trigonometrically wellbounded operators on Hilbert space. 
In the course of the exchanges during the Oberwolfach Workshop on Spectral Theory in Banach Spaces and Harmonic Analysis (July 25-31, 2004), Nigel Kalton offered the seminal suggestion that the James inequalities for super-reflexive spaces ([30]) might prove to be a useful tool for advances in spectral integration. The author wishes to thank Nigel Kalton for subsequently informing him of this perceptive viewpoint, which forms the basis for the developments below. On the heels of the Oberwolfach Workshop on Spectral Theory in Banach Spaces and Harmonic Analysis, work aimed in the direction of Kalton's suggestion was carried out in a doctoral dissertation at the University of Edinburgh [21. This thesis work and the present article spiritually overlap each other in two places, and this state of affairs will be described below in Remark 3.8 , where we discuss the anatomy of the present article's methods.

2. Background items. In this section, we recall requisite notions, starting with the basic machinery of spectral families and their associated spectral integration.

Definition 2.1. A spectral family in a Banach space $\mathfrak{X}$ is an idempotentvalued function $E(\cdot): \mathbb{R} \rightarrow \mathfrak{B}(\mathfrak{X})$ with the following properties:

(i) $E(\lambda) E(\tau)=E(\tau) E(\lambda)=E(\lambda)$ if $\lambda \leq \tau$;

(ii) $\|E\|_{u} \equiv \sup \{\|E(\lambda)\|: \lambda \in \mathbb{R}\}<\infty$;

(iii) with respect to the strong operator topology, $E(\cdot)$ is right continuous and has a left-hand limit $E\left(\lambda^{-}\right)$at each point $\lambda \in \mathbb{R}$;

(iv) $E(\lambda) \rightarrow I$ as $\lambda \rightarrow \infty$ and $E(\lambda) \rightarrow 0$ as $\lambda \rightarrow-\infty$, each limit being with respect to the strong operator topology.

If, in addition, there exist $a, b \in \mathbb{R}$ with $a \leq b$ such that $E(\lambda)=0$ for $\lambda<a$ and $E(\lambda)=I$ for $\lambda \geq b$ then $E(\cdot)$ is said to be concentrated on $[a, b]$.

Given a spectral family $E(\cdot)$ in the Banach space $\mathfrak{X}$ concentrated on a compact interval $J=[a, b]$, an associated theory of spectral integration can be developed as follows. For each bounded function $\psi: J \rightarrow \mathbb{C}$ and each partition $\mathcal{P}=\left(\lambda_{0}, \lambda_{1}, \ldots, \lambda_{n}\right)$ of $J$, where we take $\lambda_{0}=a$ and $\lambda_{n}=b$, set

$$
\mathcal{S}(\mathcal{P} ; \psi, E)=\sum_{k=1}^{n} \psi\left(\lambda_{k}\right)\left\{E\left(\lambda_{k}\right)-E\left(\lambda_{k-1}\right)\right\} .
$$

If the net $\{\mathcal{S}(\mathcal{P} ; \psi, E)\}$ converges in the strong operator topology of $\mathfrak{B}(\mathfrak{X})$ as $\mathcal{P}$ runs through the set of partitions of $J$ directed to increase by refinement, then the strong limit is called the spectral integral of $\psi$ with respect to $E(\cdot)$, and is denoted by $\int_{J} \psi(\lambda) d E(\lambda)$ or, more briefly, by $\int_{J} \psi d E$. 
In this case, we define $\int_{J}^{\oplus} \psi(\lambda) d E(\lambda)$ by writing

$$
\int_{J}^{\oplus} \psi(\lambda) d E(\lambda) \equiv \psi(a) E(a)+\int_{J} \psi(\lambda) d E(\lambda),
$$

and so $\int_{J}^{\oplus} \psi(\lambda) d E(\lambda)$ is the limit in the strong operator topology of the sums

$$
\widetilde{\mathcal{S}}(\mathcal{P} ; \psi, E) \equiv \psi(a) E(a)+\sum_{k=1}^{n} \psi\left(\lambda_{k}\right)\left\{E\left(\lambda_{k}\right)-E\left(\lambda_{k-1}\right)\right\} .
$$

It can be shown that the spectral integral $\int_{J} \psi(\lambda) d E(\lambda)$ exists for each $\psi \in \mathrm{BV}(J)$, and that the mapping

$$
\psi \mapsto \int_{J}^{\oplus} \psi(\lambda) d E(\lambda)
$$

is an identity-preserving algebra homomorphism of $\mathrm{BV}(J)$ into $\mathfrak{B}(\mathfrak{X})$ satisfying

$$
\left\|\int_{J}^{\oplus} \psi(t) d E(t)\right\| \leq\|\psi\|_{\mathrm{BV}(J)} \| \sup \{\|E(\lambda)\|: \lambda \in \mathbb{R}\},
$$

where $\|\cdot\|_{\mathrm{BV}(J)}$ denotes the usual Banach algebra norm expressed by

$$
\|\psi\|_{\mathrm{BV}(J)} \equiv \sup _{x \in J}|\psi(x)|+\operatorname{var}(\psi, J) .
$$

In this connection, we recall here a key oscillation notion for the spectral family $E(\cdot)$ in the arbitrary Banach space $\mathfrak{X}$ concentrated on a compact interval $J=[a, b]$. For each $x \in \mathfrak{X}$, and each partition of $[a, b], \mathcal{P}=(a=$ $\left.a_{0}<a_{1}<\cdots<a_{N}=b\right)$, we put

$$
\omega(\mathcal{P}, E, x)=\max _{1 \leq j \leq N} \sup \left\{\left\|E(t) x-E\left(a_{j-1}\right) x\right\|: a_{j-1} \leq t<a_{j}\right\} .
$$

Now, as $\mathcal{P}$ increases through the set of all partitions of $[a, b]$ directed to increase by refinement, we have (see Lemma 4 of [38])

$$
\lim _{\mathcal{P}} \omega(\mathcal{P}, E, x)=0 \text {. }
$$

In the setting of the arbitrary Banach space $\mathfrak{X}$, one can establish with the aid of (2.5) the following "workhorse" convergence theorem for spectral integrals of $\mathrm{BV}(J)$-functions taken with respect to $E(\cdot)$. In the setting of super-reflexive spaces, Theorems 3.9 and 3.11 below show that this convergence theorem has counterparts for functions of higher variation.

Theorem 2.2. Let $\left\{\psi_{\alpha}\right\}_{\alpha \in \mathfrak{A}}$ be a net in $\mathrm{BV}(J)$, and let $\psi$ be a complexvalued function on $J$ such that

(i) $\sup _{\alpha \in \mathfrak{A}} \operatorname{var}\left(\psi_{\alpha}, J\right)<\infty$,

(ii) $\psi_{\alpha} \rightarrow \psi$ pointwise on $J$. 


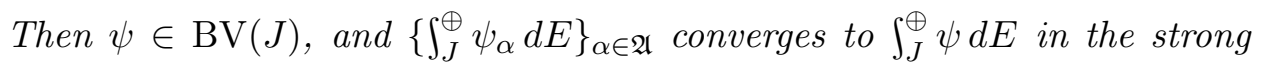
operator topology.

The foregoing basic theory of spectral integration was developed in [38]. We refer the reader to $\S 2$ of [7] for a simplified account using the above notation. We shall also consider in connection with the above matters the Banach algebra $\mathrm{BV}(\mathbb{T})$, which consists of all $\psi: \mathbb{T} \rightarrow \mathbb{C}$ such that the function $\psi^{\dagger}(t) \equiv \psi\left(e^{i t}\right)$ belongs to $\mathrm{BV}([0,2 \pi])$, furnished with the norm $\|\psi\|_{\mathrm{BV}(\mathbb{T})} \equiv\left\|\psi^{\dagger}\right\|_{\mathrm{BV}([0,2 \pi])}$. The following notation will come in handyparticularly whenever Fejér's Theorem is invoked. Given any function $f$ : $\mathbb{R} \rightarrow \mathbb{C}$ which has a right-hand limit and a left-hand limit at each point of $\mathbb{R}$, we shall denote by $f^{\#}: \mathbb{R} \rightarrow \mathbb{C}$ the function defined for every $t \in \mathbb{R}$ by putting

$$
f^{\#}(t)=\frac{1}{2}\left\{\lim _{s \rightarrow t^{+}} f(s)+\lim _{s \rightarrow t^{-}} f(s)\right\} .
$$

In the case of a function $\phi: \mathbb{T} \rightarrow \mathbb{C}$ such that $\phi\left(e^{i(\cdot)}\right): \mathbb{R} \rightarrow \mathbb{C}$ has everywhere a right-hand limit and a left-hand limit, we shall, by a slight abuse of notation, write

$$
\phi^{\#}(t)=\frac{1}{2}\left\{\lim _{s \rightarrow t^{+}} \phi\left(e^{i s}\right)+\lim _{s \rightarrow t^{-}} \phi\left(e^{i s}\right)\right\} \quad \text { for all } t \in \mathbb{R} .
$$

In particular, for each $\phi \in \mathrm{BV}(\mathbb{T})$, it is clear that we may regard the $(2 \pi)$ periodic function $\phi^{\#}$ as an element of $\mathrm{BV}(\mathbb{T})$. (In general, when there is no danger of confusion, we shall, as convenient, tacitly indulge in the conventional practice of identifying a function $\Psi$ defined on $\mathbb{T}$ with its $(2 \pi)$-periodic counterpart $\Psi\left(e^{i(\cdot)}\right)$ defined on $\mathbb{R}$.)

Definition 2.3. An operator $U \in \mathfrak{B}(\mathfrak{X})$ is said to be trigonometrically well-bounded if there is a spectral family $E(\cdot)$ in $\mathfrak{X}$ concentrated on $[0,2 \pi]$ such that $U=\int_{[0,2 \pi]}^{\oplus} e^{i \lambda} d E(\lambda)$. In this case, it is possible to arrange that $E\left((2 \pi)^{-}\right)=I$, and with this additional property the spectral family $E(\cdot)$ is uniquely determined by $U$, and is called the spectral decomposition of $U$.

REMARK 2.4. The above discussion regarding (2.3) and (2.4) shows that a trigonometrically well-bounded operator on a Banach space has a norm-continuous $\mathrm{BV}(\mathbb{T})$-functional calculus. In the setting of super-reflexive spaces, Theorem 3.7 below will extend this $\mathrm{BV}(\mathbb{T})$-functional calculus to a norm-continuous functional calculus based on functions of appropriately higher variation.

After the development in [4] of an intimately related precursor class (the "well-bounded operators of type (B)"), the class of trigonometrically well-bounded operators was introduced in [5], and its fundamental structural theory further developed in [6]. In the general Banach space setting 
(resp., in the reflexive space setting described in Proposition 1.1), trigonometrically well-bounded operators can be characterized by the precompactness in the weak operator topology (resp., the uniform boundedness) of the $(C, 1)$ means of their full set of rotated discrete ergodic Hilbert averages. (For the general Banach space case, see Theorem 5.2 of [14].) In order to discuss this recurring theme, it will be convenient to establish a notation for the sequence of trigonometric polynomials underlying it via spectral integration - specifically, for each $n \in \mathbb{N}$ and each $z \in \mathbb{T}$, we write

$$
\mathfrak{s}_{n}(z)=\sum_{0<|k| \leq n} \frac{z^{k}}{k}
$$

(thus, $\left\{\mathfrak{s}_{n}\right\}_{n=1}^{\infty}$ is the sequence of partial sums for the Fourier series of $\phi_{0} \in$ $\mathrm{BV}(\mathbb{T})$ defined by $\phi_{0}(1)=0$ and $\phi_{0}\left(e^{i t}\right)=i(\pi-t)$ for $\left.0<t<2 \pi\right)$. The fact that $\operatorname{var}\left(\mathfrak{s}_{n}, \mathbb{T}\right) \rightarrow \infty$ as $n \rightarrow \infty$ is a well-known consequence of the properties of the Lebesgue constants (see, e.g., (3.9) of [14]), and renders (2.4) incapable of bounding the sequence $\left\{\left\|\mathfrak{s}_{n}(T)\right\|\right\}_{n=1}^{\infty}$ in the case of an arbitrary trigonometrically well-bounded operator on an arbitrary Banach space $\mathfrak{X}$. The following remark guarantees that there is no way out of this, even in the setting of a general reflexive Banach space, and this fact serves to underscore the aforementioned felicitous properties which Theorem 4.3 confers on the set $\widetilde{\mathcal{W}}$ in 1.3 when the underlying Banach space is superreflexive.

REMARK 2.5. Example (3.1) in [6] exhibits a reflexive Banach space $\mathfrak{X}_{0}$ and a trigonometrically well-bounded operator $T_{0} \in \mathfrak{B}\left(\mathfrak{X}_{0}\right)$ such that for each trigonometric polynomial $Q$, we have

$$
\left\|Q\left(T_{0}\right)\right\|_{\mathfrak{B}\left(\mathfrak{X}_{0}\right)}=|Q(1)|+\operatorname{var}(Q, \mathbb{T}) .
$$

Hence $\left\|\mathfrak{s}_{n}\left(T_{0}\right)\right\|_{\mathfrak{B}_{\left(\mathfrak{X}_{0}\right)}} \rightarrow \infty$ as $n \rightarrow \infty$. A noteworthy feature of the reflexive Banach space $\mathfrak{X}_{0}$ used in this example is that, by virtue of [25] (note, e.g., Lemma 1.e.4 in [33]), $\mathfrak{X}_{0}$ cannot be made uniformly convex by equivalent renorming (in view of Corollary 3 of [26], this last can be equivalently restated by saying that the reflexive Banach space $\mathfrak{X}_{0}$ is not super-reflexive).

On a more positive note, we mention here that trigonometrically wellbounded operators do enjoy the following operator-valued variant of Fejér's Theorem (see Theorem (3.10)(i) of [7]). (For a marked improvement on the conclusion of this next theorem in the presence of super-reflexivity, see Theorem 4.4 below.)

THEOREM 2.6. Suppose that $U$ is a trigonometrically well-bounded operator on a Banach space $\mathfrak{X}$, and $E(\cdot)$ is the spectral decomposition of $U$. Let $f \in \mathrm{BV}(\mathbb{T})$, and let $f^{\#}$ be as in 2.6. Then the series $\sum_{k=-\infty}^{\infty} \widehat{f}(k) U^{k}$ is $(C, 1)$-summable in the strong operator topology to (that is, the sequence 
$\left\{\sum_{k=-n}^{n}\left(1-\frac{|k|}{n+1}\right) \widehat{f}(k) U^{k}\right\}_{n=1}^{\infty}$ converges in the strong operator topology to)

$$
\int_{0,2 \pi]}^{\oplus} f^{\#}(t) d E(t)
$$

The centerpiece of our considerations in $\$ 3$ will be a proof that, in the context of super-reflexivity, spectral integration against $E(\cdot)$ can be extended from $\mathrm{BV}(\mathbb{T})$ to the broader classes $V_{p}(\mathbb{T})$ consisting of the functions of bounded $p$-variation, where $p$ ranges over an appropriate subinterval of $(1, \infty)$ (see Theorem 3.7 below). To avoid later digressions, we take up here the definition of the $p$-variation of a function $\psi$.

Definition 2.7. Let $J=[a, b]$ be a compact interval of $\mathbb{R}$. For $1 \leq p<\infty$, the $p$-variation of a function $\psi: J \rightarrow \mathbb{C}$ is specified by writing

$$
\operatorname{var}_{p}(\psi,[a, b])=\sup \left\{\sum_{k=1}^{N}\left|\psi\left(x_{k}\right)-\psi\left(x_{k-1}\right)\right|^{p}\right\}^{1 / p}
$$

where the supremum is extended over all partitions $a=x_{0}<x_{1}<\cdots<$ $x_{N}=b$ of $[a, b]$.

By definition, the class $V_{p}(J)$ consists of all functions $\psi: J \rightarrow \mathbb{C}$ such that $\operatorname{var}_{p}(\psi,[a, b])<\infty$. It is readily verified that $V_{p}(J)$ becomes a unital Banach algebra under pointwise operations when endowed with the norm $\|\cdot\|_{V_{p}(J)}$ specified by

$$
\|\psi\|_{V_{p}(J)}=\sup \{|\psi(x)|: x \in J\}+\operatorname{var}_{p}(\psi, J) .
$$

Moreover, if $\psi \in V_{p}(J)$, then $\lim _{x \rightarrow y^{+}} \psi(x)$ exists for each $y \in[a, b)$ and $\lim _{x \rightarrow y^{-}} \psi(x)$ exists for each $y \in(a, b]$, and the set of discontinuities of $\psi$ in $J$ is countable. It is elementary that $V_{1}(J)$ and $\mathrm{BV}(J)$ consist of the same functions, and also that $V_{q}(J) \subseteq V_{r}(J)$ when $1 \leq q \leq r<\infty$, since $\|\psi\|_{V_{p}(J)}$ is a decreasing function of $p$. For additional fundamental features of $V_{p}(J)$, see, e.g., $\S 2$ in [11].

For $\psi: \mathbb{T} \rightarrow \mathbb{C}$, we define $\operatorname{var}_{p}(\psi, \mathbb{T})$ to $\operatorname{be~}_{\operatorname{var}_{p}}\left(\psi\left(e^{i(\cdot)}\right),[0,2 \pi]\right)$, and we designate by $V_{p}(\mathbb{T})$ the class consisting of all functions $\psi: \mathbb{T} \rightarrow \mathbb{C}$ such that $\operatorname{var}_{p}(\psi, \mathbb{T})<\infty$. With pointwise operations on $\mathbb{T}, V_{p}(\mathbb{T})$ likewise becomes a unital Banach algebra when furnished with the norm

$$
\|\psi\|_{V_{p}(\mathbb{T})}=\left\|\psi\left(e^{i(\cdot)}\right)\right\|_{V_{p}([0,2 \pi])}=\sup \{|\psi(z)|: z \in \mathbb{T}\}+\operatorname{var}_{p}(\psi, \mathbb{T}) .
$$

REMARK 2.8. (i) For $1 \leq p<\infty$ and $\psi: \mathbb{T} \rightarrow C$, there is also a rotation-invariant notion for the $p$-variation of $\psi$ on $\mathbb{T}$, which serves as an alternative to $\operatorname{var}_{p}(\psi, \mathbb{T})$ defined above. Specifically, we can define

$$
\mathfrak{v}_{p}(\psi, \mathbb{T})=\sup \left\{\sum_{k=1}^{N}\left|\psi\left(e^{i t_{k}}\right)-\psi\left(e^{i t_{k-1}}\right)\right|^{p}\right\}^{1 / p},
$$


where the supremum is taken over all finite sequences $-\infty<t_{0}<t_{1}<\cdots<$ $t_{N}=t_{0}+2 \pi<\infty$. It is evident that

$$
\operatorname{var}_{p}(\psi, \mathbb{T}) \leq \mathfrak{v}_{p}(\psi, \mathbb{T}) \leq 2 \operatorname{var}_{p}(\psi, \mathbb{T}),
$$

and that $\mathfrak{v}_{1}(\psi, \mathbb{T})=\operatorname{var}_{1}(\psi, \mathbb{T})$. Moreover, for $1 \leq p<\infty, V_{p}(\mathbb{T})$ is also a unital Banach algebra under the norm $\|\cdot\|_{\mathfrak{v}_{p}(\mathbb{T})}$ given by

$$
\|\psi\|_{\mathfrak{v}_{p}(\mathbb{T})}=\sup \{|\psi(z)|: z \in \mathbb{T}\}+\mathfrak{v}_{p}(\psi, \mathbb{T}),
$$

which, by virtue of (2.8), is obviously equivalent to the Banach algebra norm $\|\cdot\|_{V_{p}(\mathbb{T})}$ defined above. (When convenient, we shall use the equivalence of the norms $\|\cdot\|_{\mathfrak{v}_{p}(\mathbb{T})}$ and $\|\cdot\|_{V_{p}(\mathbb{T})}$ without comment.) Straightforward application of the Generalized Minkowski Inequality shows that if $F \in L^{1}(\mathbb{T})$ and $\psi \in V_{p}(\mathbb{T})$, then the convolution $F * \psi$ belongs to $V_{p}(\mathbb{T})$, with

$$
\|F * \psi\|_{V_{p}(\mathbb{T})} \leq\|F\|_{L^{1}(\mathbb{T})}\|\psi\|_{\mathfrak{v}_{p}(\mathbb{T})} \leq 2\|F\|_{L^{1}(\mathbb{T})}\|\psi\|_{V_{p}(\mathbb{T})} .
$$

(ii) It is worth noting here that if $1<q<\infty$, then $\bigcup_{1 \leq p<q} V_{p}(\mathbb{T})$ is not dense in $V_{q}(\mathbb{T})$. To see this, first note that if $1 \leq p<\infty$ and $f \in V_{p}(\mathbb{T})$, then, in the notation of [29] we have, $f \in \Lambda_{p}$. This is a standard inclusion, established for $p=1$ in Lemma 9 of [29], and for $1<p<\infty$ on pages 259, 260 of [40] (nowadays this inclusion for $1<p<\infty$ is also transparent via, e.g., Theorem 3.1 of [23]). Hence Lemma 11 of [29] shows that $\{\widehat{f}(k)\}_{k=-\infty}^{\infty}$, the sequence of Fourier coefficients of $f$, satisfies

$$
\sup \left\{|k|^{1 / p}|\widehat{f}(k)|: k \in \mathbb{Z}\right\}<\infty .
$$

In view of this, we can define for $1 \leq p<\infty$ the linear mapping $\mathfrak{T}_{p}$ : $V_{p}(\mathbb{T}) \rightarrow \ell^{\infty}(\mathbb{Z})$ by writing $\mathfrak{T}_{p}(f)=\left\{|k|^{1 / p} \widehat{f}(k)\right\}_{k=-\infty}^{\infty}$. It follows via the Closed Graph Theorem that $\mathfrak{T}_{p}$ is continuous, and so the following set $\mathcal{N}_{p}(\mathbb{T})$, which coincides with $\left(\mathfrak{T}_{p}\right)^{-1}\left(c_{0}(\mathbb{Z})\right)$, is a closed subspace of $V_{p}(\mathbb{T})$ :

$$
\mathcal{N}_{p}(\mathbb{T})=\left\{g \in V_{p}(\mathbb{T}):|k|^{1 / p} \widehat{g}(k) \rightarrow 0 \text { as }|k| \rightarrow \infty\right\} .
$$

It is clear from 2.10 that $\bigcup_{1 \leq p<q} V_{p}(\mathbb{T}) \subseteq \mathcal{N}_{q}(\mathbb{T})$. However, $F_{q}$, Hardy's $(2 \pi)$-periodic, Weierstrass-type, continuous, nowhere differentiable function from [28, which is specified by

$$
F_{q}(t)=\sum_{n=0}^{\infty} 2^{-n / q} \cos \left(2^{n} t\right) \quad \text { for all } t \in \mathbb{R},
$$

belongs to $\operatorname{Lip}_{1 / q}(\mathbb{R})$ by 1.33 of $[28]$, and hence its restriction $F_{q} \mid[0,2 \pi]$ can be regarded as belonging to $V_{q}(\mathbb{T})$. It is clear that for each non-negative integer $n$,

$$
2^{n / q} \widehat{F_{q}}\left(2^{n}\right)=\frac{1}{2}
$$

whence $F_{q} \mid[0,2 \pi]$ does not belong to $\mathcal{N}_{q}(\mathbb{T})$. (Compare (9.4) of [40].) 
If we replace absolute values by norms in the foregoing definitions of $p$-variation, we arrive at the corresponding definitions for vector-valued functions. Furthermore, for a vector-valued function $f$ defined on $\mathbb{R}$ (including the scalar-valued case), the standard counterpart for $\mathbb{R}$ of $p$-variation is given by

$$
\operatorname{var}_{p}(f, \mathbb{R})=\sup _{-\infty<a<b<\infty} \operatorname{var}_{p}(f,[a, b]) .
$$

If $E(\cdot)$ is a spectral family of projections in an arbitrary Banach space $\mathfrak{X}$,

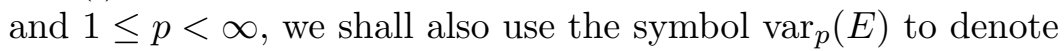

$$
\sup \left\{\operatorname{var}_{p}(E(\cdot) x, \mathbb{R}):\|x\| \leq 1\right\} \text {. }
$$

3. Super-reflexivity and spectral integration of $V_{p}(\mathbb{T})$ with $p>1$. For extensive details and terminology regarding the structure theory of super-reflexive spaces, we refer the interested reader to, e.g., Part 4 of [2]. One of R. C. James' inequalities for super-reflexive spaces (Theorem 3 of [30]) states the following.

TheOREM 3.1. Let $X$ be a super-reflexive Banach space. If $\phi$ and $K$ are real numbers such that

$$
0<2 \phi<1 / K \leq 1,
$$

then there is $q=q(X, \phi, K) \in(1, \infty)$ such that for any normalized basic sequence $\left\{y_{j}\right\}$ in $X$ with basis constant not exceeding $K$, we have

$$
\phi\left\{\sum_{j}\left|a_{j}\right|^{q}\right\}^{1 / q} \leq\left\|\sum_{j} a_{j} y_{j}\right\|,
$$

for all scalar sequences $\left\{a_{j}\right\}$ such that $\sum_{j} a_{j} y_{j}$ converges.

In the context of a spectral family of projections in a super-reflexive space, James's Theorem 3.1 above readily specializes so as to take the following form.

Proposition 3.2. If $E(\cdot)$ is a spectral family of projections in a superreflexive Banach space $X$, and $\phi$ is a real number satisfying

$$
0<\phi<\frac{1}{4\|E\|_{u}}
$$

then there is a real number $q=q\left(X, \phi,\|E\|_{u}\right) \in(1, \infty)$ such that

$$
\operatorname{var}_{q}(E) \leq \frac{2\|E\|_{u}}{\phi}
$$

Proof. Let $x \in X \backslash\{0\}$, and suppose that $-\infty<\lambda_{0}<\lambda_{1}<\cdots<$ $\lambda_{N}<\infty$. Let $\left\{z_{j}\right\}_{j=1}^{M}$ be the basic sequence consisting of all non-zero terms extracted from $\left\{\left\{E\left(\lambda_{k}\right)-E\left(\lambda_{k-1}\right)\right\} x\right\}_{k=1}^{N}$, let $\left\{y_{j}\right\}_{j=1}^{M}$ be the normalized basic sequence $\left\{z_{j} /\left\|z_{j}\right\|\right\}_{j=1}^{M}$ (whose basis constant clearly does not exceed 
$\left.2\|E\|_{u}\right)$, and let $\left\{a_{j}\right\}_{j=1}^{M}$ be the sequence of real numbers $\left\{\left\|z_{j}\right\|\right\}_{j=1}^{M}$. Then, in the present context, (3.1) becomes the desired conclusion $(3.3)$, since the sum in the majorant of (3.1) telescopes here.

Since we shall not require any specificity for the roles played by the constants $\phi,\|E\|_{u}$, and $q=q\left(X, \phi,\|E\|_{u}\right)$ in Proposition 3.2, we include here the following condensed version (which can also be derived directly from Proposition IV.II.3 on pages 249-250 of [2] by similar reasoning to that above after using the equivalent renorming of $X$ specified by $\|x\| \equiv$ $\sup _{-\infty<a<b<\infty}\|\{E(b)-E(a)\} x\|$ to convert to a monotone basic sequence).

Proposition 3.3. If $E(\cdot)$ is a spectral family of projections in a superreflexive Banach space $X$, then there is a constant $q \in(1, \infty)$ such that $\operatorname{var}_{q}(E)<\infty$.

The obvious vehicle for using Proposition 3.3 to derive the spectral integration of $V_{p}(\mathbb{T})$ for appropriate values of $p \in(1, \infty)$ is the following fundamental theorem of Young-Stieltjes integration (see $\S 10$ of [40]).

Theorem 3.4. Suppose that $J=[a, b]$ is a compact interval, $1<p, q$ $<\infty, p^{-1}+q^{-1}>1$, and $f \in V_{p}(J), g \in V_{q}(J)$ have no common discontinuities. Then the Riemann-Stieltjes integral $\int_{a}^{b} f(t) d g(t)$ exists and obeys the estimate

$$
\left|\int_{a}^{b} f(t) d g(t)\right| \leq\left\{1+\zeta\left(\frac{1}{p}+\frac{1}{q}\right)\right\}\|f\|_{V_{p}(J)} \operatorname{var}_{q}(g, J) .
$$

(Here $\zeta$ designates the Riemann zeta function specified by $\zeta(s)=\sum_{n=1}^{\infty} n^{-s}$ for $s>1$.)

THeOREM 3.5. Let $X$ be a super-reflexive Banach space, and let $E(\cdot)$ be the spectral decomposition of a trigonometrically well-bounded operator $U \in \mathfrak{B}(X)$. Let $q \in(1, \infty)$ be the index furnished for $E(\cdot)$ by Proposition 3.3 so that $\operatorname{var}_{q}(E)<\infty$. Let $u \in\left(1, q^{\prime}\right)$, where $q^{\prime}=q(q-1)^{-1}$ is the conjugate index of $q$. Then, in terms of the notation of (2.6), for every $f \in \mathrm{BV}(\mathbb{T})$ we have

$$
\left\|\int_{[0,2 \pi]}^{\oplus} f^{\#}(t) d E(t)\right\| \leq 3\left\{1+\zeta\left(\frac{1}{u}+\frac{1}{q}\right)\right\}\|f\|_{V_{u}(\mathbb{T})} \operatorname{var}_{q}(E) .
$$

Proof. Here and henceforth we denote by $\left\{\kappa_{n}\right\}_{n=0}^{\infty}$ the Fejér kernel for $\mathbb{T}$,

$$
\kappa_{n}(z) \equiv \sum_{k=-n}^{n}\left(1-\frac{|k|}{n+1}\right) z^{k}
$$

Clearly $u^{-1}+q^{-1}>1$. For $f \in \mathrm{BV}(\mathbb{T})$, each trigonometric polynomial $\kappa_{n} * f$ 
is in $\mathrm{BV}(\mathbb{T}) \subseteq V_{u}(\mathbb{T})$, with

$$
\left\|\kappa_{n} * f\right\|_{\mathrm{BV}(\mathbb{T})} \leq\|f\|_{\mathrm{BV}(\mathbb{T})} .
$$

For the integral

$$
\int_{[0,2 \pi]}\left(\kappa_{n} * f\right)\left(e^{i t}\right) d x^{*}(E(t) x)
$$

(which automatically exists for arbitrary $x \in X$, and $x^{*}$ in the dual space $X^{*}$, we now apply Theorem 3.4 to the pair of functions $\kappa_{n} * f \in V_{u}(\mathbb{T})$ and $x^{*}(E(\cdot) x) \in V_{q}([0,2 \pi])$ to obtain the estimate

$$
\begin{aligned}
\mid \int_{[0,2 \pi]}\left(\kappa_{n} * f\right)\left(e^{i t}\right) & d x^{*}(E(t) x) \mid \\
\leq & \left\{1+\zeta\left(\frac{1}{u}+\frac{1}{q}\right)\right\}\left\|\kappa_{n} * f\right\|_{V_{u}(\mathbb{T})} \operatorname{var}_{q}(E)\|x\|\left\|x^{*}\right\|,
\end{aligned}
$$

and consequently for each $n$, we see with the aid of this last estimate that

$$
\begin{aligned}
\| \int_{[0,2 \pi]}\left(\kappa_{n} * f\right)\left(e^{i t}\right) d E(t) & \\
& \leq\left\{1+\zeta\left(\frac{1}{u}+\frac{1}{q}\right)\right\}\left\|\kappa_{n} * f\right\|_{V_{u}(\mathbb{T})} \operatorname{var}_{q}(E) \\
& \leq 2\left\{1+\zeta\left(\frac{1}{u}+\frac{1}{q}\right)\right\}\|f\|_{V_{u}(\mathbb{T})} \operatorname{var}_{q}(E) .
\end{aligned}
$$

Since $\left\{\kappa_{n} * f\right\}_{n=0}^{\infty}$ converges pointwise to $f^{\#}$ on $\mathbb{T}$ while its terms have uniformly bounded 1-variations, we can infer via Theorem 2.2 above that, in the strong operator topology,

$$
\int_{[0,2 \pi]}\left(\kappa_{n} * f\right)\left(e^{i t}\right) d E(t) \rightarrow \int_{[0,2 \pi]} f^{\#}(t) d E(t) .
$$

Hence (3.5) shows that (3.4) holds.

In order to pass from the estimate in (3.4) for the spectral integral of $f^{\#}$ when $f \in \mathrm{BV}(\mathbb{T})$ to the spectral integration of $V_{p}(\mathbb{T})$-functions, we shall need to rely on the following exemplar of the tools which spectral integration furnishes for such situations.

TheOREM 3.6. Suppose that $U$ is a trigonometrically well-bounded operator on an arbitrary Banach space $\mathfrak{X}, E(\cdot)$ is the spectral decomposition of $U$, and $1<u<\infty$. Suppose further that there is a constant $\tau$ such that

$$
\left\|\int_{[0,2 \pi]}^{\oplus} \psi^{\#}\left(e^{i t}\right) d E(t)\right\| \leq \tau\|\psi\|_{V_{u}(\mathbb{T})} \quad \text { for all } \psi \in \mathrm{BV}(\mathbb{T}) .
$$

Then if $1 \leq p<u$, the spectral integral $\int_{[0,2 \pi]} \phi\left(e^{i t}\right) d E(t)$ exists for each $\phi \in V_{p}(\mathbb{T})$, and the mapping $\phi \in V_{p}(\mathbb{T}) \mapsto \int_{[0,2 \pi]}^{\oplus} \phi\left(e^{i t}\right) d E(t)$ is an identity- 
preserving algebra homomorphism of $V_{p}(\mathbb{T})$ into $\mathfrak{B}(\mathfrak{X})$ such that

$$
\left\|\int_{[0,2 \pi]}^{\oplus} \phi\left(e^{i t}\right) d E(t)\right\| \leq \tau K_{p, u}\|\phi\|_{V_{p}(\mathbb{T})} \quad \text { for all } \phi \in V_{p}(\mathbb{T}) .
$$

Proof. A demonstration of the current theorem can readily be modeled after the proof of Theorem 2.1 in [11] by replacing the Fourier multiplier norm estimate in Proposition 2.3 et seq. of [11] by the present hypothesis (3.6). Alternatively, one can extract key elements of a proof for the current theorem by making suitable modifications to the reasoning for its Marcinkiewicz power-classes counterpart in Theorem 12 of [18].

By taking $u=2^{-1}\left(p+q^{\prime}\right)$ in Theorem 3.5 while combining Theorems 3.5 and 3.6 we arrive at the following principal result, which guarantees spectral integration of $V_{p}(\mathbb{T})$ spaces in the presence of super-reflexivity, and thereby extends to each $V_{p}(\mathbb{T})$ space, throughout an appropriate range of $p>1$, the $\mathrm{BV}(\mathbb{T})$-functional calculus for trigonometrically well-bounded operators.

TheOREM 3.7. Let $X$ be a super-reflexive Banach space, and let $E(\cdot)$ be the spectral decomposition of a trigonometrically well-bounded operator $U \in \mathfrak{B}(X)$. Let $q \in(1, \infty)$ be the index furnished for $E(\cdot)$ by Proposition 3.3 so that $\operatorname{var}_{q}(E)<\infty$. Let $p \in\left(1, q^{\prime}\right)$, where $q^{\prime}=q(q-1)^{-1}$ is the conjugate index of $q$. Then the spectral integral $\int_{[0,2 \pi]} \phi\left(e^{i t}\right) d E(t)$ exists for each $\phi \in V_{p}(\mathbb{T})$, and the mapping $\phi \in V_{p}(\mathbb{T}) \mapsto \int_{[0,2 \pi]}^{\oplus} \phi\left(e^{i t}\right) d E(t)$ is an identity-preserving algebra homomorphism of $V_{p}(\mathbb{T})$ into $\mathfrak{B}(X)$ such that

$$
\left\|\int_{[0,2 \pi]}^{\oplus} \phi\left(e^{i t}\right) d E(t)\right\| \leq K_{p, q} \operatorname{var}_{q}(E)\|\phi\|_{V_{p}(\mathbb{T})} \quad \text { for all } \phi \in V_{p}(\mathbb{T}) .
$$

REMARK 3.8. (i) As already indicated above, from both a conceptual and historical standpoint, Proposition 3.2 (along with its abbreviated version in Proposition 3.3 can best be viewed as the immediate specialization to spectral families of James' celebrated estimate for super-reflexive spaces here quoted as Theorem 3.1. On the basis of extensive calculations aided by [30], Theorem 2.1 of [21] asserts what amounts to Proposition 3.2 above. The reasoning devoted to Theorem 2.1 in [21] occurs there on pp. 14-28, 31, with the following description on page 23: "The proof of Theorem 2.1 is rather involved, and requires several technical results".

(ii) Some generic spectral integration tool for the general Banach space setting, such as Theorem 3.6, seems to be required for the transition from Proposition 3.2 and the fundamental theorem of Young-Stieltjes integration reproduced in Theorem 3.4 in order to arrive at Theorem 3.7. The reasoning offered for Theorem 4.1 in [21], which purports to establish the same result as Theorem 3.7 above without such a transitional tool, is flawed, primarily 
because it rests on the false premise that $V_{1}(\mathbb{T})$ is norm-dense in $V_{p}(\mathbb{T})$, if $1<p<\infty$, in contradiction to the result in Remark 2.8(ii) above.

We now proceed to associate with Theorem 3.7 a useful convergence theorem for appropriate nets of spectral integrals in the context of superreflexivity. This (as well as Theorem 3.11 below) furnishes the promised extension of Theorem 2.2 to functions of higher variation.

TheOREm 3.9. Assume the hypotheses on $X, E(\cdot), U$, and $q$ of Theorem 3.7, and let $p \in\left(1, q^{\prime}\right)$. Suppose that $\left\{g_{\beta}\right\}_{\beta \in B}$ is a net of mappings from $\mathbb{T}$ into $\mathbb{C}$ satisfying

$$
\rho \equiv \sup \left\{\operatorname{var}_{p}\left(g_{\beta}, \mathbb{T}\right): \beta \in B\right\}<\infty,
$$

and such that for each $\beta \in B$, and each $t_{0} \in \mathbb{R}$,

$$
\lim _{t \rightarrow t_{0}-} g_{\beta}\left(e^{i t}\right)=g_{\beta}\left(e^{i t_{0}}\right) .
$$

Suppose further that $\left\{g_{\beta}\right\}_{\beta \in B}$ converges pointwise on $\mathbb{T}$ to a complex-valued function $g$. Then $g \in V_{p}(\mathbb{T})$, and the net

$$
\left\{\int_{[0,2 \pi]}^{\oplus} g_{\beta}\left(e^{i t}\right) d E(t)\right\}_{\beta \in B}
$$

converges in the strong operator topology of $\mathfrak{B}(X)$ to $\int_{[0,2 \pi]}^{\oplus} g\left(e^{i t}\right) d E(t)$.

Proof. Clearly, $\operatorname{var}_{p}(g, \mathbb{T}) \leq \rho<\infty$. Choose $q_{1}$ so that $1<q<q_{1}<\infty$ and $p^{-1}+q_{1}^{-1}>1$. Fix $x \in X \backslash\{0\}$, let $\varepsilon>0$ be given, and use 2.5 to infer that $[0,2 \pi]$ has a partition $\mathcal{P}_{\varepsilon}=\left(0=t_{0}<t_{1}<\cdots<t_{J}=2 \pi\right)$ such that

$$
\omega(\mathcal{U}, E, x)<\varepsilon \quad \text { for any refinement } \mathcal{U} \text { of } \mathcal{P}_{\varepsilon} .
$$

For an arbitrary pair of refinements of $\mathcal{P}_{\varepsilon}$, say $\mathcal{P}=\left(0=a_{0}<a_{1}<\cdots<\right.$ $\left.a_{N}=2 \pi\right), \mathcal{Q}=\left(0=b_{0}<b_{1}<\cdots<b_{M}=2 \pi\right)$, and for any $\beta \in B$, we shall now consider the following two sums:

$$
\begin{aligned}
S_{1} & \equiv \sum_{j=1}^{N} E\left(a_{j-1}\right) x\left\{g_{\beta}\left(e^{i a_{j}}\right)-g_{\beta}\left(e^{i a_{j-1}}\right)\right\}, \\
S_{2} & \equiv \sum_{m=1}^{M} E\left(b_{m-1}\right) x\left\{g_{\beta}\left(e^{i b_{m}}\right)-g_{\beta}\left(e^{i b_{m-1}}\right)\right\} .
\end{aligned}
$$

For $1 \leq \nu \leq J$, let $I_{\nu}=\left[y_{\nu}, z_{\nu}\right]$ be the rightmost subinterval of $\mathcal{P}$ contained in the subinterval $\left[t_{\nu-1}, t_{\nu}\right]$ of $\mathcal{P}_{\varepsilon}$, and let $S_{1}^{\prime}$ denote the sum $S_{1}$ after the replacement of the terms $E\left(y_{\nu}\right) x\left\{g_{\beta}\left(e^{i z_{\nu}}\right)-g_{\beta}\left(e^{i y_{\nu}}\right)\right\}, 1 \leq \nu \leq J$, by corresponding terms $E\left(y_{\nu}\right) x\left\{g_{\beta}\left(e^{i z_{\nu}^{\prime}}\right)-g_{\beta}\left(e^{i y_{\nu}}\right)\right\}$, where $y_{\nu}<z_{\nu}^{\prime}<z_{\nu}, 1 \leq \nu \leq J$. Moreover, we can choose these points $z_{\nu}^{\prime}, 1 \leq \nu \leq J$, so that we can similarly form $S_{2}^{\prime}$ from $S_{2}$ by truncating to the same right end-point $z_{\nu}^{\prime}$ the rightmost 
in the string of subintervals of $\mathcal{Q}$ contained in each $\left[t_{\nu-1}, t_{\nu}\right]$. In terms of this notation, we can write

$$
S_{1}^{\prime}-S_{2}^{\prime}=\sum_{\nu=1}^{J}\left(\Omega_{\nu}-\Lambda_{\nu}\right),
$$

where, for $1 \leq \nu \leq J, \Omega_{\nu}$ (resp., $\Lambda_{\nu}$ ) represents the contribution to $S_{1}^{\prime}$ (resp., $S_{2}^{\prime}$ ) of the string of intervals that are contained in the subinterval $\left[t_{\nu-1}, t_{\nu}\right]$ of $\mathcal{P}_{\varepsilon}$. Provided that the pair of reciprocal indices involved has sum exceeding 1 (as is true here for $q_{1}^{-1}, p^{-1}$ ), the reasoning leading up to and including Young's estimate (6.4) in [40] can be applied to any pair of qualifying functions such that one is vector-valued, and the other is scalar-valued (a quick way to see this is to apply temporarily an arbitrary continuous linear functional, then invoke directly the results in [40] for a pair of scalar-valued functions, and then revert to norms in the ultimate vector-valued expressions).

Applying Young's estimate (6.4), and then the technique in (10.8) of [40], together with (3.9) above, we can infer that for $1 \leq \nu \leq J$ we have, in terms of the Riemann zeta function $\zeta$,

$$
\left\|\Omega_{\nu}-\Lambda_{\nu}\right\|
$$

$\leq 2(2 \varepsilon)^{\left(q_{1}-q\right) / q_{1}}\left\{1+\zeta\left(q_{1}^{-1}+p^{-1}\right)\right\} \operatorname{var}_{q}^{q / q_{1}}\left(E(\cdot) x,\left[t_{\nu-1}, t_{\nu}\right]\right) \operatorname{var}_{p}\left(g_{\beta},\left[t_{\nu-1}, t_{\nu}\right]\right)$.

Summing the estimates in (3.10) from $\nu=1$ to $J$, and then applying Hölder's inequality (for the pair of indices $q_{1}, p$ ) to the resulting majorant, we find that

$$
\left\|S_{1}^{\prime}-S_{2}^{\prime}\right\| \leq 2(2 \varepsilon)^{\left(q_{1}-q\right) / q_{1}}\left\{1+\zeta\left(q_{1}^{-1}+p^{-1}\right)\right\} \operatorname{var}_{q}^{q / q_{1}}(E(\cdot) x,[0,2 \pi]) \operatorname{var}_{p}\left(g_{\beta}, \mathbb{T}\right) .
$$

If in the sums $S_{1}^{\prime}$ and $S_{2}^{\prime}$ we now let each $z_{\nu}^{\prime}$ approach from the left the corresponding point $t_{\nu}$, then $(3.8)$ gives

$$
\begin{array}{r}
\left\|\sum_{j=1}^{N} E\left(a_{j-1}\right) x\left\{g_{\beta}\left(e^{i a_{j}}\right)-g_{\beta}\left(e^{i a_{j-1}}\right)\right\}-\sum_{m=1}^{M} E\left(b_{m-1}\right) x\left\{g_{\beta}\left(e^{i b_{m}}\right)-g_{\beta}\left(e^{i b_{m-1}}\right)\right\}\right\| \\
\leq 2(2 \varepsilon)^{\left(q_{1}-q\right) / q_{1}}\left\{1+\zeta\left(q_{1}^{-1}+p^{-1}\right)\right\} \operatorname{var}_{q}^{q / q_{1}}(E(\cdot) x,[0,2 \pi]) \rho .
\end{array}
$$

For notational convenience, let us denote by $\delta_{\varepsilon}$ the majorant in $(3.12)$, while keeping in mind that $\delta_{\varepsilon} \rightarrow 0$ as $\varepsilon \rightarrow 0^{+}$. After a summation by parts is performed on each of the vector-valued sums appearing in the minorant of (3.12), we find that, in the notation of 2.2 , the estimate 3.12 can be rewritten as follows:

$$
\left\|\widetilde{\mathcal{S}}\left(\mathcal{P} ; g_{\beta}\left(e^{i(\cdot)}\right), E\right) x-\widetilde{\mathcal{S}}\left(\mathcal{Q} ; g_{\beta}\left(e^{i(\cdot)}\right), E\right) x\right\| \leq \delta_{\varepsilon} .
$$


Upon letting $\mathcal{P}$ run through all refinements of $\mathcal{P}_{\varepsilon}$ in $(3.13)$, while simultaneously holding fixed both the arbitrary refinement $\mathcal{Q}$ of $\mathcal{P}_{\varepsilon}$ and the arbitrary $\beta \in B$, we get

$$
\left\|\int_{[0,2 \pi]}^{\oplus} g_{\beta}\left(e^{i t}\right) d E(t) x-\widetilde{\mathcal{S}}\left(\mathcal{Q} ; g_{\beta}\left(e^{i(\cdot)}\right), E\right) x\right\| \leq \delta_{\varepsilon} .
$$

Next, while holding $\mathcal{P}, \mathcal{Q}$ fixed in (3.13), we let $\beta$ run through $B$ to obtain, via the pointwise convergence on $\mathbb{T}$,

$$
\left\|\widetilde{\mathcal{S}}\left(\mathcal{P} ; g\left(e^{i(\cdot)}\right), E\right) x-\widetilde{\mathcal{S}}\left(\mathcal{Q} ; g\left(e^{i(\cdot)}\right), E\right) x\right\| \leq \delta_{\varepsilon} .
$$

Letting $\mathcal{P}$ run through all refinements of $\mathcal{P}_{\varepsilon}$ in this yields, for every refinement $\mathcal{Q}$ of $\mathcal{P}_{\varepsilon}$,

$$
\left\|\int_{[0,2 \pi]}^{\oplus} g\left(e^{i t}\right) d E(t) x-\widetilde{\mathcal{S}}\left(\mathcal{Q} ; g\left(e^{i(\cdot)}\right), E\right) x\right\| \leq \delta_{\varepsilon} .
$$

Combining this estimate with (3.14), we find that for all $\beta \in B$, and every refinement $\mathcal{Q}$ of $\mathcal{P}_{\varepsilon}$,

$$
\begin{aligned}
\left\|\int_{[0,2 \pi]}^{\oplus} g_{\beta}\left(e^{i t}\right) d E(t) x-\int_{[0,2 \pi]}^{\oplus} g\left(e^{i t}\right) d E(t) x\right\| \\
\leq 2 \delta_{\varepsilon}+\left\|\widetilde{\mathcal{S}}\left(\mathcal{Q} ; g_{\beta}\left(e^{i(\cdot)}\right), E\right) x-\widetilde{\mathcal{S}}\left(\mathcal{Q} ; g\left(e^{i(\cdot)}\right), E\right) x\right\| .
\end{aligned}
$$

In (3.15), we now specialize $\mathcal{Q}$ to be $\mathcal{P}_{\varepsilon}$, and we see from the pointwise convergence of $\left\{g_{\beta}\right\}_{\beta \in B}$ to $g$ on $\mathbb{T}$ that for all sufficiently large $\beta \in B$,

$$
\left\|\int_{[0,2 \pi]}^{\oplus} g_{\beta}\left(e^{i t}\right) d E(t) x-\int_{[0,2 \pi]}^{\oplus} g\left(e^{i t}\right) d E(t) x\right\| \leq 3 \delta_{\varepsilon} \cdot
$$

REMARK 3.10. Our treatment of the spectral integration of functions of higher variation emphasizes applications thereof to a unified framework of trigonometrically well-bounded operators and related periodic functions. For this purpose $[0,2 \pi]$ conveniently serves as the fundamental interval. It is worth noting, however, that the above Theorems 3.7 and 3.9 do not need to be tied directly to trigonometrically well-bounded operators, since they readily imply their analogues for spectral families concentrated on arbitrary intervals by using simple affine changes of the real variable (e.g., mapping $[0, \pi]$ onto an interval $J=[a, b])$. The outcome, which includes an extension of the $\mathrm{BV}(J)$-functional calculus induced by spectral families $(2.3)$, can be stated as follows.

THEOREM 3.11. Let $E(\cdot)$ be a spectral family of projections in a superreflexive Banach space $X$. Suppose that $E(\cdot)$ is concentrated on a compact interval $J=[a, b]$, and let $q \in(1, \infty)$ be the index furnished for $E(\cdot)$ by Proposition 3.3 so that $\operatorname{var}_{q}(E)<\infty$. Let $p \in\left(1, q^{\prime}\right)$. Then the spectral integral $\int_{J} \Phi d E$ exists for each $\Phi \in V_{p}(J)$, and the mapping $\Phi \in V_{p}(J) \mapsto$ 
$\int_{J}^{\oplus} \Phi d E$ is a continuous identity-preserving homomorphism of the Banach algebra $V_{p}(J)$ into the Banach algebra $\mathfrak{B}(X)$ such that

$$
\left\|\int_{J}^{\oplus} \Phi d E\right\| \leq K_{p, q} \operatorname{var}_{q}(E)\|\Phi\|_{V_{p}(J)} \quad \text { for all } \Phi \in V_{p}(J) .
$$

If $\left\{\Phi_{\beta}\right\}_{\beta \in B}$ is a net of mappings from $J$ into $\mathbb{C}$ satisfying

$$
\sup \left\{\operatorname{var}_{p}\left(\Phi_{\beta}, J\right): \beta \in B\right\}<\infty,
$$

and such that for each $\beta \in B$, and each $t_{0} \in(a, b]$,

$$
\lim _{t \rightarrow t_{0}-} \Phi_{\beta}(t)=\Phi_{\beta}\left(t_{0}\right),
$$

and if $\left\{\Phi_{\beta}\right\}_{\beta \in B}$ converges pointwise on $J$ to a complex-valued function $\Phi$, then $\Phi \in V_{p}(J)$, and the net

$$
\left\{\int_{J}^{\oplus} \Phi_{\beta} d E\right\}_{\beta \in B}
$$

converges in the strong operator topology of $\mathfrak{B}(X)$ to $\int_{J}^{\oplus} \Phi d E$.

4. Some consequences. The stage is almost set for the main result of this section (Theorem 4.3), which will establish the precompactness relative to the strong operator topology of the set of rotated Hilbert averages $\widetilde{\mathcal{W}}$ corresponding to a trigonometrically well-bounded operator $U$ on a super-reflexive space. In order to obtain this result, we shall also require the following two auxiliary items from the literature.

Proposition 4.1. Suppose that $1 \leq p<\infty$. Then we have, for the sequence of trigonometric polynomials $\left\{\mathfrak{s}_{n}\right\}_{n=1}^{\infty}$ in (2.7),

$$
\sup _{n \in \mathbb{N}} \operatorname{var}_{p}\left(\mathfrak{s}_{n}, \mathbb{T}\right)<\infty \quad \text { if and only if } p>1 \text {. }
$$

Proof. Since, as was noted in conjunction with (2.7), $\operatorname{var}_{1}\left(\mathfrak{s}_{n}, \mathbb{T}\right) \rightarrow \infty$ as $n \rightarrow \infty$, it suffices to have

$$
\sup _{n \in \mathbb{N}} \operatorname{var}_{p}\left(\mathfrak{s}_{n}, \mathbb{T}\right)<\infty \quad \text { if } p>1 .
$$

The derivation of this is included in $\S 12$ of the article [40].

In view of this, the set $\mathfrak{S}$ consisting of all rotates of $\left\{\mathfrak{s}_{n}: n \in \mathbb{N}\right\}$ must satisfy

$$
\sup _{n \in \mathbb{N}, z \in \mathbb{T}}\left\|\mathfrak{s}_{n}((\cdot) z)\right\|_{V_{p}(\mathbb{T})}<\infty \quad \text { if } p>1,
$$

by virtue of 2.8 , and because $\left\{\mathfrak{s}_{n}\right\}_{n=1}^{\infty}$ is the sequence of partial sums for the Fourier series of a $B V(\mathbb{T})$-function, whence

$$
\sup _{n \in \mathbb{N}}\left\|\mathfrak{s}_{n}\right\|_{L^{\infty}(\mathbb{T})}<\infty .
$$


The second auxiliary item we shall rely on is the following convenient formulation of the "Helly Selection Theorem for Functions of Bounded $p$ Variation" (Theorem 2.4 of [36]). (Although it will not be an issue for us, we note that in the parlance of [36], the symbol $\operatorname{var}_{p}$ denotes what is, in the sense of our notation, $\operatorname{var}_{p}^{p}$.)

THEOREM 4.2. Let $\mathcal{F}$ be a sequence of functions mapping a subset $\mathcal{M}$ of $\mathbb{R}$ to a metric space $\mathfrak{Y}$, and such that, for some $p \in[1, \infty), \mathcal{F}$ has uniformly bounded p-variation on $\mathcal{M}$ (in symbols, $\left.\sup \left\{\operatorname{var}_{p}(F, \mathcal{M}): F \in \mathcal{F}\right\}<\infty\right)$. Suppose further that for each $t \in \mathcal{M},\{F(t): F \in \mathcal{F}\}$ has compact closure in $\mathfrak{Y}$. Then $\mathcal{F}$ has a subsequence $\left\{f_{n}\right\}_{n=1}^{\infty}$ pointwise convergent on $\mathcal{M}$ to a function $f: \mathcal{M} \rightarrow \mathfrak{Y}$ such that

$$
\operatorname{var}_{p}(f, \mathcal{M}) \leq \sup \left\{\operatorname{var}_{p}(F, \mathcal{M}): F \in \mathcal{F}\right\}<\infty .
$$

THEOREM 4.3. If $U$ is a trigonometrically well-bounded operator on a super-reflexive Banach space $X$, then the closure, relative to the strong operator topology, of the class $\widetilde{\mathcal{W}}$ specified in 1.3 by

$$
\widetilde{\mathcal{W}}=\left\{\sum_{0<|k| \leq n} \frac{z^{k}}{k} U^{k}: n \in \mathbb{N}, z \in \mathbb{T}\right\}
$$

is compact in the strong operator topology, and hence, in particular,

$$
\sup \{\|T\|: T \in \widetilde{\mathcal{W}}\}<\infty .
$$

Conversely, if $\mathfrak{X}_{0}$ is a reflexive Banach space, and $U \in \mathfrak{B}\left(\mathfrak{X}_{0}\right)$ is an invertible operator such that (4.4) holds, then $U$ is trigonometrically well-bounded.

Proof. Let $E(\cdot)$ be the spectral decomposition of $U$, and choose $q, p$ as in the hypotheses of Theorem 3.7. Let $x \in X \backslash\{0\}$. We are required to show that the set $\widetilde{\mathcal{W}} x$ is totally bounded in the metric space defined by the norm of $X$. For this purpose, let $\mathcal{G}$ be a sequence in $\widetilde{\mathcal{W}} x$. Hence for some sequence $\mathcal{F}$ taken from the set of trigonometric polynomials $\mathfrak{S}$ appearing in the minorant of $(4.2)$, we can express $\mathcal{G}$ as $\mathcal{F}(U) x$. By virtue of $(4.2)$ and Theorem 4.2, we can extract from the sequence of trigonometric polynomials $\mathcal{F}$ a subsequence $\left\{f_{k}\right\}_{k=1}^{\infty}$ pointwise convergent on $\mathbb{T}$ to a function $f: \mathbb{T} \rightarrow \mathbb{C}$ such that

$$
\operatorname{var}_{p}(f, \mathbb{T}) \leq \sup \left\{\operatorname{var}_{p}(F, \mathbb{T}): F \in \mathfrak{S}\right\}<\infty .
$$

By Theorem 3.9, applied to $\left\{f_{k}\right\}_{k=1}^{\infty}$, we see that $\left\{f_{k}(U)\right\}_{k=1}^{\infty}$ converges in the strong operator topology to $\int_{[0,2 \pi]}^{\oplus} f\left(e^{i t}\right) d E(t)$.

The converse conclusion follows directly from Proposition 1.1, since for each $z \in \mathbb{T}$, the $(C, 1)$ averages appearing in 1.2$)$ are the means of the corresponding discrete Hilbert averages in 4.3.

An application of Theorem 3.7 of [12] to 4.4 yields the following improvement of Theorem 2.6 . 
TheOREM 4.4. Let $X$ be a super-reflexive Banach space, let $U \in \mathfrak{B}(X)$ be trigonometrically well-bounded, and let $E(\cdot)$ be the spectral decomposition of $U$. Then for each $f \in \mathrm{BV}(\mathbb{T})$, the series $\sum_{k=-\infty}^{\infty} \widehat{f}(k) U^{k}$ converges in the strong operator topology to $\int_{[0,2 \pi]}^{\oplus} f^{\#}(t) d E(t)$.

In the presence of super-reflexivity, we now also have the following extension of Theorem 2.6 from $\mathrm{BV}(\mathbb{T})$ to spaces $V_{p}(\mathbb{T})$, for appropriate $p>1$.

Theorem 4.5. Let $X$ be a super-reflexive Banach space, and let $U \in$ $\mathfrak{B}(X)$ be a trigonometrically well-bounded operator. Denote by $E(\cdot)$ the spectral decomposition of $U$, let $q \in(1, \infty)$ be the index furnished for $E(\cdot)$ by Proposition 3.3 so that $\operatorname{var}_{q}(E)<\infty$, and let $p \in\left(1, q^{\prime}\right)$. If $\phi \in V_{p}(\mathbb{T})$, then for each $x \in X$,

$$
\left\|\sum_{\nu=-n}^{n}\left(1-\frac{|\nu|}{n+1}\right) \widehat{\phi}(\nu) U^{\nu} x-\left\{\int_{[0,2 \pi]}^{\oplus} \phi^{\#}(t) d E(t)\right\} x\right\| \rightarrow 0 \text { as } n \rightarrow \infty .
$$

Proof. Clearly, the sequence of trigonometric polynomials $\left\{\kappa_{n} * \phi\right\}_{n \geq 0}$ has the property that $\sup _{n \geq 0}\left\|\kappa_{n} * \phi\right\|_{V_{p}(\mathbb{T})}<\infty$, and by Fejér's Theorem, $\left(\kappa_{n} * \phi\right)\left(e^{i t}\right) \rightarrow \phi^{\#}(t)$ for all $t \in \mathbb{R}$. The desired conclusion is now an immediate consequence of Theorem 3.9 applied to the pointwise convergent sequence $\left\{\kappa_{n} * \phi\right\}_{n \geq 0}$.

REMARK 4.6. In contrast to the situation for BV(T)-functions in Theorem 4.4, it is an open question whether or not one can, for the general $\phi \in V_{p}(\mathbb{T})$, improve the strong $(C, 1)$-convergence in 4.5$)$ to strong convergence of the series $\sum_{\nu=-\infty}^{\infty} \widehat{\phi}(\nu) U^{\nu}$. In this regard, one can use Theorem 3.1 of [37] in combination with Theorem 4.5 to obtain the following partial result in the positive direction. We omit the details for expository reasons.

Proposition 4.7. Suppose that $\mathfrak{Y}$ is a UMD space having an unconditional basis, let $U \in \mathfrak{B}(\mathfrak{Y})$ be a trigonometrically well-bounded operator. Denote by $E(\cdot)$ the spectral decomposition of $U$. Let $q \in(1, \infty)$ be the index furnished for $E(\cdot)$ by Proposition 3.3 so that $\operatorname{var}_{q}(E)<\infty$, and let $p \in\left(1, q^{\prime}\right)$. If $\phi \in V_{p}(\mathbb{T})$, then for each $y \in \mathfrak{Y}$ we have, for almost all $z \in \mathbb{T}$,

$$
\left\|\left(\sum_{k=-n}^{n} \widehat{\phi}(k) U^{k} z^{k}\right) y-\left(\int_{[0,2 \pi]}^{\oplus}\left(\phi_{z}\right)^{\#}(t) d E(t)\right) y\right\|_{\mathfrak{Y}} \rightarrow 0 \quad \text { as } n \rightarrow \infty .
$$

REMARK 4.8. Since the Haar system is an unconditional basis for $L^{r}([0,1]), 1<r<\infty$, the space $L^{r}(\mathbb{T})$ satisfies the hypotheses on $\mathfrak{Y}$ of Proposition 4.7. In particular, by specializing to the value $r=2$, we see that any separable Hilbert space (finite-dimensional or infinite-dimensional) satisfies these hypotheses on $\mathfrak{Y}$. 


\section{Operator-weighted Hilbert sequence spaces and trigonomet-} rically well-bounded shift operators. Henceforth, $\mathfrak{K}$ will be an arbitrary Hilbert space with inner product $\langle\cdot, \cdot\rangle$. As shown in Theorem 2.3 of [16], shifts on appropriate operator-weighted Hilbert sequence spaces serve as a model for the general behavior of trigonometrically well-bounded operators on arbitrary Hilbert spaces. More specifically, to any invertible operator $V \in \mathfrak{B}(\mathfrak{K})$ there correspond a bilateral operator-valued weight sequence $\mathfrak{W}_{V} \subseteq \mathfrak{B}(\mathfrak{K})$ and an affiliated Hilbert sequence space $\ell^{2}\left(\mathfrak{W}_{V}\right)$ such that $V$ is trigonometrically well-bounded on $\mathfrak{K}$ if and only if the right bilateral shift $\mathcal{R}$ is a trigonometrically well-bounded operator on $\ell^{2}\left(\mathfrak{W}_{V}\right)$; moreover, if this is the case, then the norm properties of trigonometric polynomials of $\mathcal{R}$ mirror the norm properties of trigonometric polynomials of $V$. (See $(5.6)$ below. For additional background facts regarding these matters, see [12].) In this section, we shall discuss how application of the preceding sections to this circle of ideas in Hilbert space affords some new insights into the role of the Hilbert transform and of multiplier theory in non-commutative analysis.

We begin by describing the relevant class of operator-weighted Hilbert sequence spaces. An operator-valued weight sequence on $\mathfrak{K}$ will be a bilateral sequence $\mathfrak{W}=\left\{W_{k}\right\}_{k=-\infty}^{\infty} \subseteq \mathfrak{B}(\mathfrak{K})$ such that for each $k \in \mathbb{Z}, W_{k}$ is a positive, invertible, self-adjoint operator. We associate with $\mathfrak{W}$ the weighted Hilbert space $\ell^{2}(\mathfrak{W})$ consisting of all sequences $x=\left\{x_{k}\right\}_{k=-\infty}^{\infty} \subseteq \mathfrak{K}$ such that

$$
\sum_{k=-\infty}^{\infty}\left\langle W_{k} x_{k}, x_{k}\right\rangle<\infty,
$$

and furnished with the inner product $\langle\langle\cdot, \cdot\rangle\rangle$ specified by

$$
\langle\langle x, y\rangle\rangle=\sum_{k=-\infty}^{\infty}\left\langle W_{k} x_{k}, y_{k}\right\rangle .
$$

Thus, $\ell^{2}(\mathfrak{W})$ is a generalization to non-commutative analysis of the $\ell^{2}$-spaces defined by scalar-valued weight sequences in the special case where $\mathfrak{K}=\mathbb{C}$. (For the continuous variable generalization from scalar-valued weights to operator-valued weights, see [39].) Note that for each $z \in \mathbb{T}$, there is a natural unitary operator $\Delta_{z}$ defined on $\ell^{2}(\mathfrak{W})$ by writing $\Delta_{z}\left(\left\{x_{k}\right\}_{k=-\infty}^{\infty}\right)=$ $\left\{z^{k} x_{k}\right\}_{k=-\infty}^{\infty}$.

The links between the considerations of the previous sections and $\ell^{2}(\mathfrak{W})$ stem from the interplay between $\ell^{2}(\mathfrak{W})$ and the discrete Hilbert kernel $h$ : $\mathbb{Z} \rightarrow \mathbb{R}$, which, in terms of the function $\phi_{0} \in \mathrm{BV}(\mathbb{T})$ specified in conjunction with $(2.7)$, is expressed by $h=\widehat{\phi_{0}}$. Thus $h(0)=0$, and $h(k)=k^{-1}$ for $k \in \mathbb{Z} \backslash\{0\}$. The truncates $\left\{h_{N}\right\}_{N=1}^{\infty}$ of the discrete Hilbert kernel $h$ are defined by writing, for each $N \in \mathbb{N}$ and each $k \in \mathbb{Z}, h_{N}(k)=h(k)$ if $|k| \leq N$, and $h_{N}(k)=0$ if $|k|>N$. The formal operator of convolution by 
$h$ on $\ell^{2}(\mathfrak{W})$ will be referred to as the discrete Hilbert transform, and will be symbolized by $D$ (convolution by $h_{N}$ on $\ell^{2}(\mathfrak{W})$ will be denoted by $\left.D_{N}\right)$. If $h$ defines a bounded convolution operator from $\ell^{2}(\mathfrak{W})$ into $\ell^{2}(\mathfrak{W})$, we shall say that $\mathfrak{W}$ possesses the Treil-Volberg property. It was shown in [12] that in the context of $\ell^{2}(\mathfrak{W})$, one can define an operator-valued counterpart (the discrete analogue of [39]) for the Muckenhoupt $A_{2}$-weight condition-if this condition is satisfied by $\mathfrak{W}$, we write $\mathfrak{W} \in A_{2}(\mathfrak{K})$. Since we do not need this $A_{2}(\mathfrak{K})$ weight condition for our present considerations, we shall not pursue it further, except to note that the condition $\mathfrak{W} \in A_{2}(\mathfrak{K})$ is always necessary, but, for the continuous-variable case and infinite-dimensional $\mathfrak{K}$, is known not to be sufficient, for $\mathfrak{W}$ to possess the Treil-Volberg property (see, respectively, Proposition 4.4 of [12, and Theorem 1.1 of [27]).

The connection between the Treil-Volberg property and the right (bilateral) shift $\mathcal{R}: \ell^{2}(\mathfrak{W}) \rightarrow \mathfrak{K}^{\mathbb{Z}}$ specified by

$$
\mathcal{R}\left(\left\{x_{k}\right\}_{k=-\infty}^{\infty}\right)=\left\{x_{k-1}\right\}_{k=-\infty}^{\infty}
$$

is expressed as follows (Theorem 4.12 of [12]).

Proposition 5.1. Let $\mathfrak{W}=\left\{W_{k}\right\}_{k=-\infty}^{\infty}$ be an operator-valued weight sequence on the arbitrary Hilbert space $\mathfrak{K}$. Then the following assertions are equivalent:

(i) W has the Treil-Volberg property.

(ii) The right shift $\mathcal{R}$ is a trigonometrically well-bounded operator on $\ell^{2}(\mathfrak{W})$.

(iii) $\mathcal{R}$ is a bounded invertible operator on $\ell^{2}(\mathfrak{W})$ such that

$$
\sup _{n \in \mathbb{N}}\left\|\sum_{0<|k| \leq n}\left(1-\frac{|k|}{n+1}\right) \frac{\mathcal{R}^{k}}{k}\right\|<\infty .
$$

REMARK 5.2. If $\mathcal{R} \in \mathfrak{B}\left(\ell^{2}(\mathfrak{W})\right)$, then for each $z \in \mathbb{T}, \Delta_{z} \mathcal{R} \Delta_{\bar{z}}=z \mathcal{R}$, and hence the condition 1.2 reduces to $(5.1)$ in the context of Proposition 5.1 (iii).

By virtue of (4.4), we can add the following two conditions to the list of equivalent conditions in Proposition 5.1.

Proposition 5.3. Under the hypotheses of Proposition 5.1, each of the following two conditions is equivalent to the conditions (i)-(iii) listed therein:

(iv) $\mathcal{R}$ is a bounded invertible operator on $\ell^{2}(\mathfrak{W})$ such that

$$
\sup _{n \in \mathbb{N}}\left\|\sum_{0<|k| \leq n} \frac{\mathcal{R}^{k}}{k}\right\|<\infty .
$$


(v) $\left\{D_{N}\right\}_{N=1}^{\infty} \subseteq \mathfrak{B}\left(\ell^{2}(\mathfrak{W})\right)$, with

$$
\sup _{N \in \mathbb{N}}\left\|D_{N}\right\|_{\mathfrak{B}\left(\ell^{2}(\mathfrak{W})\right)}<\infty .
$$

Proof. It is elementary that (iv) $\Rightarrow$ (iii). The implication (ii) $\Rightarrow$ (iv) is a consequence of 4.4. If (iv) holds, then for each $N \in \mathbb{N}$,

$$
\mathfrak{s}_{N}(\mathcal{R})=D_{N},
$$

and hence (v) holds. So the proof of Proposition 5.3 boils down to assuming (v) in order to show any one of the conditions (i) through (iv). Since there is no a priori reason to infer from $(\mathrm{v})$ that $\mathcal{R}$ is a bounded invertible operator on $\ell^{2}(\mathfrak{W})$, we cannot make immediate use of (5.4), and so we shall sidestep this difficulty by establishing (i) directly. Since the Hilbert space $\ell^{2}(\mathfrak{W})$ is, in particular, reflexive, it follows from (5.3) that the closure of

$$
\mathfrak{D} \equiv\left\{D_{N}: N \in N\right\}
$$

in the weak operator topology of $\mathfrak{B}\left(\ell^{2}(\mathfrak{W})\right)$ is compact in the weak operator topology of $\mathfrak{B}\left(\ell^{2}(\mathfrak{W})\right)$. Consequently, there are a subnet $\left\{D_{N_{\gamma}}\right\}_{\gamma \in \Gamma}$ and an operator $\mathfrak{H} \in \mathfrak{B}\left(\ell^{2}(\mathfrak{W})\right)$ such that

$$
D_{N_{\gamma}} \rightarrow \mathfrak{H} \quad \text { in the weak operator topology of } \mathfrak{B}\left(\ell^{2}(\mathfrak{W})\right) \text {. }
$$

Hence it will suffice to verify that for every vector $y=\left\{y_{k}\right\}_{k=-\infty}^{\infty} \in \ell^{2}(\mathfrak{W})$ such that the support of $y$ is a singleton, $\mathfrak{H}$ acts on $y$ as convolution by $h$. It is a routine matter to perform this verification by using (5.5) in conjunction with such vectors.

REMARK 5.4. In classical single-variable Fourier analysis, as well as in its generalizations to norm inequalities involving scalar-valued weights, the boundedness of the relevant Hilbert transform goes hand-in-hand with the boundedness of pillars like the Hardy-Littlewood maximal function and the maximal Hilbert transform - which leave in their wake the uniform boundedness of the Hilbert transform's truncates. This familiar scenario ultimately entails the validity of the relevant version of the Marcinkiewicz Multiplier Theorem and of the Littlewood-Paley Theorem. However, in the framework of condition (i) of Proposition 5.1 such underpinnings as maximal operators are lacking, and moreover, Theorem 6.1 of [16] shows that there is an operator-valued weight sequence $\mathfrak{W}_{0}$ on the Hilbert space $\ell^{2}(\mathbb{N})$ such that $\mathfrak{W}_{0}$ enjoys the Treil-Volberg property, but the analogues of the classical Marcinkiewicz Multiplier Theorem and the Littlewood-Paley Theorem fail to hold on $\ell^{2}\left(\mathfrak{W}_{0}\right)$. One motivation for obtaining the above implication $(\mathrm{i}) \Rightarrow(\mathrm{v})$ is that it, nevertheless, confirms the survival of the uniform boundedness for the Hilbert transform's truncates, in an environment where so many mainstays fail to carry over. The next theorem adds still more to the 
positive side of the ledger by extending this type of boundedness result to appropriate function classes.

TheOREM 5.5. Suppose that $\mathfrak{K}$ is an arbitrary Hilbert space, and $\mathfrak{W}=$ $\left\{W_{k}\right\}_{k=-\infty}^{\infty}$ is an operator-valued weight sequence on $\mathfrak{K}$ having the TreilVolberg property. Then there is $\gamma \in(1, \infty)$ such that for each $p$ satisfying $1 \leq p<\gamma$, and each function $\phi \in V_{p}(\mathbb{T})$, convolution by the inverse Fourier transform $\phi^{\vee}$ on $\ell^{2}(\mathfrak{W})$ is a bounded linear mapping $\mathfrak{F}_{\phi}$ of $\ell^{2}(\mathfrak{W})$ into $\ell^{2}(\mathfrak{W})$ satisfying

$$
\left\|\mathfrak{F}_{\phi}\right\|_{\mathfrak{B}\left(\ell^{2}(\mathfrak{W})\right)} \leq K_{\mathfrak{W}, p}\|\phi\|_{V_{p}(\mathbb{T})}
$$

Proof. Combine Theorems 4.2 and 4.3 of [16] and Corollary 4.4 of [16] with Theorem 3.7 above.

We finish this section with a brief sketch of how the above scene furnishes a model for estimates with trigonometrically well-bounded operators on Hilbert spaces. Suppose that $V \in \mathfrak{B}(\mathfrak{K})$ is an invertible operator, and let $\mathfrak{W}_{V}$ be the operator-valued weight sequence on the Hilbert space $\mathfrak{K}$ given by $\mathfrak{W}_{V}=\left\{\left(V^{k}\right)^{*} V^{k}\right\}_{k=-\infty}^{\infty}$. Lemma 2.2 of [16] and Theorem 2.3 of [16] guarantee that the right shift $\mathcal{R}$ is a bounded invertible linear mapping of $\ell^{2}\left(\mathfrak{W}_{V}\right)$ onto itself such that for every trigonometric polynomial $Q$,

$$
\|Q(\mathcal{R})\|_{\mathfrak{B}\left(\ell^{2}\left(\mathfrak{W}_{V}\right)\right)}=\sup _{z \in \mathbb{T}}\|Q(z V)\|_{\mathfrak{B}(\mathfrak{K})} .
$$

In view of Proposition 1.1 and the equivalence of conditions (ii) and (iii) in Proposition 5.1, it follows directly from (5.6) that the right shift $\mathcal{R}$ is trigonometrically well-bounded on $\ell^{2}\left(\mathfrak{W}_{V}\right)$ if and only if $V$ is trigonometrically well-bounded on $\mathfrak{K}$.

\section{References}

[1] D. J. Aldous, Unconditional bases and martingales in $L_{p}(F)$, Math. Proc. Cambridge Philos. Soc. 85 (1979), 117-123.

[2] B. Beauzamy, Introduction to Banach Spaces and Their Geometry, North-Holland Math. Stud. 68 (Notas de Mat. 86), Elsevier Science, New York, 1982.

[3] E. Berkson, J. Bourgain, and T. A. Gillespie, On the almost everywhere convergence of ergodic averages for power-bounded operators on $L^{p}$-subspaces, Integral Equations Operator Theory 14 (1991), 678-715.

[4] E. Berkson and H. R. Dowson, On uniquely decomposable well-bounded operators, Proc. London Math. Soc. (3) 22 (1971), 339-358.

[5] E. Berkson and T. A. Gillespie, AC functions on the circle and spectral families, J. Operator Theory 13 (1985), 33-47.

[6] —, - Fourier series criteria for operator decomposability, Integral Equations Operator Theory 9 (1986), 767-789.

[7] —, 一, Stečkin's theorem, transference, and spectral decompositions, J. Funct. Anal. 70 (1987), 140-170. 
[8] E. Berkson and T. A. Gillespie, The spectral decomposition of weighted shifts and the $A_{p}$ condition, Colloq. Math. (special volume dedicated to A. Zygmund) 60-61 (1990), 507-518.

[9] - - -, Spectral decompositions and harmonic analysis on UMD spaces, Studia Math. 112 (1994), 13-49.

[10] —, -, Mean-boundedness and Littlewood-Paley for separation-preserving operators, Trans. Amer. Math. Soc. 349 (1997), 1169-1189.

[11] —, - , The q-variation of functions and spectral integration of Fourier multipliers, Duke Math. J. 88 (1997), 103-132.

[12] —, - Mean $_{2}$-bounded operators on Hilbert space and weight sequences of positive operators, Positivity 3 (1999), 101-133.

[13] - - -, Spectral integration from dominated ergodic estimates, Illinois J. Math. 43 (1999), 500-519.

[14] —, 一, Spectral decompositions, ergodic averages, and the Hilbert transform, Studia Math. 144 (2001), 39-61.

[15] —, 一, A Tauberian theorem for ergodic averages, spectral decomposability, and the dominated ergodic estimate for positive invertible operators, Positivity 7 (2003), $161-175$.

[16] —, - Shifts as models for spectral decomposability on Hilbert space, J. Operator Theory 50 (2003), 77-106.

[17] - - - Operator means and spectral integration of Fourier multipliers, Houston J. Math. 30 (2004), 767-814.

[18] -, - , The q-variation of functions and spectral integration from dominated ergodic estimates, J. Fourier Anal. Appl. 10 (2004), 149-177.

[19] - - - An $\mathfrak{M}_{q}(\mathbb{T})$-functional calculus for power-bounded operators on certain UMD spaces, Studia Math. 167 (2005), 245-257.

[20] E. Berkson, T. A. Gillespie, and P. S. Muhly, Abstract spectral decompositions guaranteed by the Hilbert transform, Proc. London Math. Soc. (3) 53 (1986), 489-517.

[21] D. Blagojevic, Spectral families and geometry of Banach spaces, PhD thesis, Univ. of Edinburgh, 2007; http://www.era.lib.ed.ac.uk/handle/1842/2389.

[22] J. Bourgain, Some remarks on Banach spaces in which martingale difference sequences are unconditional, Ark. Mat. 21 (1983), 163-168.

[23] V. V. Chistyakov and O. E. Galkin, On maps of bounded p-variation with $p>1$, Positivity 2 (1998), 19-45.

[24] R. Coifman, J. L. Rubio de Francia, et S. Semmes, Multiplicateurs de Fourier de $L^{p}(\mathbb{R})$ et estimations quadratiques, C. R. Acad. Sci. Paris Sér. I Math. 306 (1988), 351-354.

[25] M. M. Day, Reflexive Banach spaces not isomorphic to uniformly convex spaces, Bull. Amer. Math. Soc. 47 (1941), 313-317.

[26] P. Enflo, Banach spaces which can be given an equivalent uniformly convex norm, Israel J. Math. 13 (1972), 281-288.

[27] T. A. Gillespie, S. Pott, S. Treil, and A. Volberg, Logarithmic growth for weighted Hilbert transforms and vector Hankel operators, J. Operator Theory 52 (2004), 103112 .

[28] G. H. Hardy, Weierstrass's non-differentiable function, Trans. Amer. Math. Soc. 17 (1916), 301-325.

[29] G. H. Hardy and J. E. Littlewood, A convergence criterion for Fourier series, Math. Z. 28 (1928), 612-634.

[30] R. C. James, Super-reflexive spaces with bases, Pacific J. Math. 41 (1972), 409-419.

[31] —, Super-reflexive Banach spaces, Canad. J. Math. 24 (1972), 896-904. 
[32] Y. Katznelson, An Introduction to Harmonic Analysis, Dover, New York, 1976.

[33] J. Lindenstrauss and L. Tzafriri, Classical Banach Spaces II: Function Spaces, Ergeb. Math. Grenzgeb. 97, Springer, New York, 1979.

[34] B. Maurey, Système de Haar, in: Séminaire Maurey-Schwartz 1974-1975, Centre Math. École Polytechnique, Paris, 1975, 26 pp.

[35] G. Pisier, Un exemple concernant la super-réflexivité, ibid., 12 pp.

[36] J. E. Porter, Helly's selection principle for functions of bounded p-variation, Rocky Mountain J. Math. 35 (2005), 675-679.

[37] J. L. Rubio de Francia, Fourier series and Hilbert transforms with values in UMD Banach spaces, Studia Math. 81 (1985), 95-105.

[38] P. G. Spain, On well-bounded operators of type (B), Proc. Edinburgh Math. Soc. (2) 18 (1972), 35-48.

[39] S. Treil and A. Volberg, Wavelets and the angle between past and future, J. Funct. Anal. 143 (1997), 269-308.

[40] L. C. Young, An inequality of the Hölder type, connected with Stieltjes integration, Acta Math. 67 (1936), 251-282.

Earl Berkson

Department of Mathematics

University of Illinois

1409 W. Green Street

Urbana, IL 61801 U.S.A.

E-mail: berkson@math.uiuc.edu

Received January 30, 2010

Revised version July 7, 2010 